\title{
Acoustic feedback neutralization in active noise control systems
}

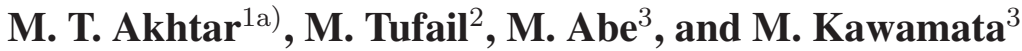 \\ ${ }^{1}$ Department of Electrical Engineering, UAE University, P.O.Box 17555, Al-Ain, \\ $U A E$ \\ ${ }^{2}$ Department of Electrical Engineering, Pakistan Institute of Engineering and \\ Applied Sciences, Islamabad, Pakistan \\ ${ }^{3}$ Graduate School of Engineering, Tohoku University, Sendai 980-8579, JAPAN \\ a)akhtar@ieee.org
}

\begin{abstract}
This letter proposes a method for active noise control (ANC) systems with online acoustic feedback neutralization. The proposed method is a modified version of authors' previous work, and the objective here is to reduce the computational complexity. This is achieved by combining the role of feedback path modeling and neutralization filters. The computer simulations are carried out which demonstrate that the proposed method achieves better performance than the existing methods and at somewhat reduced computational cost.
\end{abstract}

Keywords: active noise control, FIR adaptive algorithms, acoustic feedback-path modeling.

Classification: Science and engineering for electronics

\section{References}

[1] S. M. Kuo and D. R. Morgan, Active Noise Control Systems-Algorithms and DSP Implementation, New York: Wiley, 1996.

[2] S. M. Kuo and D. R. Morgan, "Active Noise Control: A tutorial review," Proc. IEEE, vol. 87, no. 6, pp. 943-973, June 1999.

[3] B. Widrow and S. D. Stearns, Adaptive Signal Processing, Prentice Hall, New Jersey, 1985.

[4] L. A. Poole, G. E. Warnaka, and R. C. Cutter, "The implementation of digital filters using a modified Widrow-Hoff algorithm for the adaptive cancellation of acoustic noise," in Proc. ISCAS, 1984, pp. 21.7.1-4.

[5] S. M. Kuo and J. Luan, "On-Line Modeling and Feedback Compensation for Multiple-Channel Active Noise Control Systems," Applied Signal Processing, vol. 1, no. 2, pp. 64-75, 1994.

[6] S. M. Kuo, "Active Noise Control System and Method for On-Line Feedback Path Modeling," US Patent 6,418,227, July 9, 2002.

[7] M. T. Akhtar, M. Abe, and M. Kawamata, "On Active Noise Control Systems With Online Acoustic Feedback Path Modeling," IEEE Trans. Audio Speech Language Processing, vol. 15, no. 2, pp. 593-600, Feb. 2007. 


\section{Introduction}

A block diagram of a single channel feedforward active noise control (ANC) systems [1] is shown in Fig. 1. Here $P(z)$ is the primary path between the noise source and the error microphone, $S(z)$ is the secondary path between canceling loudspeaker and error microphone, $F(z)$ is the feedback path from canceling loudspeaker to the reference microphone, and filtered-x LMS (FxLMS) algorithm $[2,3]$ is used to adapt the ANC adaptive filter $W(z)$. The ANC system uses the reference microphone to pick up the reference noise $x(n)$, processes this input with an adaptive filter to generate an antinoise $y(n)$ to cancel primary noise acoustically in the duct, and uses an error microphone to measure the error $e(n)$ and to update the adaptive filter coefficients. Unfortunately, a loudspeaker on a duct wall will generate plane waves propagating both upstream and downstream. Therefore, the antinoise output to the loudspeaker not only cancels noise downstream, but also radiates upstream to the reference microphone, resulting in a corrupted reference signal $x(n)$. This coupling of acoustic waves from secondary loudspeaker to the reference microphone is called acoustic feedback.

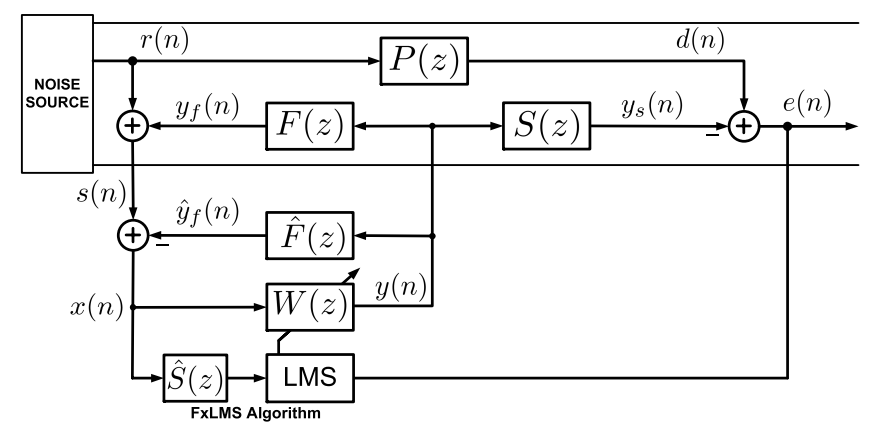

Fig. 1. Block diagram of single-channel ANC system with fixed feedback neutralization.

Consider Fig. 1, and assuming that the feedback path neutralization (FBPN) filter $\hat{F}(z)$ is not present, the error signal $z$-transform is expressed as

$$
E(z)=P(z) R(z)-S(z) Y(z)=P(z) R(z)-S(z) \frac{W(z) R(z)}{1-W(z) F(z)} .
$$

The convergence of $W(z)$ means (ideally) $E(z)=0$. This requires $W(z)$ to converge to the following solution:

$$
W^{\circ}(z)=\frac{P(z)}{S(z)+P(z) F(z)} .
$$

This simple analysis shows that due to acoustic feedback the ANC system will be unstable, if the coefficients of $W(z)$ are large enough so that $W(z) F(z)=1$ at some frequency.

The simplest approach to solving the feedback problem is to use a separate FBPN filter with in the controller, as shown in Fig. 1. This electrical model of 
the feedback path is driven by the secondary source control signal, $y(n)$, and its output is subtracted from the reference sensor signal. The FBPN filter, $\hat{F}(z)$, may be obtained offline prior to the operation of ANC system when the reference noise $r(n)$ does not exist. In many practical cases, however, $r(n)$ always exists, and $F(z)$ may be time varying. For these cases, online modeling of $F(z)$ is needed to ensure the convergence of the FxLMS algorithm for ANC systems.

Broadly speaking there are two types of signal processing methods for adaptive feedback neutralization: 1) IIR-filter based methods, and 2) FIRfilter based methods. In IIR based structures, the stability cannot be guaranteed, and the adaptation may converge to a local minimum. Consequently, only the FIR-based methods are examined further in this paper. A detailed review of existing FIR-filter-based methods $[4,5,6]$ for online FBPM can be found in [7]. In this paper, we describe only the best existing method proposed by Kuo in [6], identify its problems, and then describe the proposed method in comparison with the authors' previous work of [7]. The main objective is to achieve improved performance at reduced computational complexity. The rest of the paper is organized as follows. Section 2 presents the details of the proposed method. Section 3 details the simulations results and presents concluding remarks.

\section{ANC Systems with Online Feedback Path Modeling}

The Kuo's method [6] is shown in Fig. 2 (a). Here the noise source is assumed to be predictable. The feedback path modeling (FBPM) is achieved by using an additive-random noise based adaptive filter, $\hat{F}(z)$. The weights of FBPM filter, $\hat{F}(z)$, are copied to the FBPN filter, $\hat{F}(z)$, taking $y(n)$ as input. A discrimination filter, based on a decorrelation delay, removes the predictable component from the desired response of the FBPM filter. This method achieves improved online FBPM, however, there are a few problems: 1) the performance depends on the proper choice of the decorrelation delay, and 2) it works only for predictable noise sources. To overcome these problems, in [7] authors have proposed a method using adaptive noise cancelation (ADNC) filter in place of the discrimination filter in [6]. The main advantage of this method is that it can work for both predictable and un-predictable noise sources. Furthermore, the method does not use decorrelation delay. In these two methods, two filters are used for $\hat{F}(z)$ : adaptive for FBPM and fixed for FBPN. Here we combine these two tasks of modeling and neutralization in one adaptive filter, and call it as feedback path modeling and neutralization (FBPMN) filter.

\subsection{Proposed Method}

The proposed method is shown in Fig. 2 (b). As in previous methods [5, 6, 7], a random noise signal $v(n)$, uncorrelated with the reference signal $x(n)$ is added with the output $y(n)$ of $W(z)$. The sum $[y(n)+v(n)]$ is propagated by the canceling loudspeaker. The signal propagates downstream to generate 


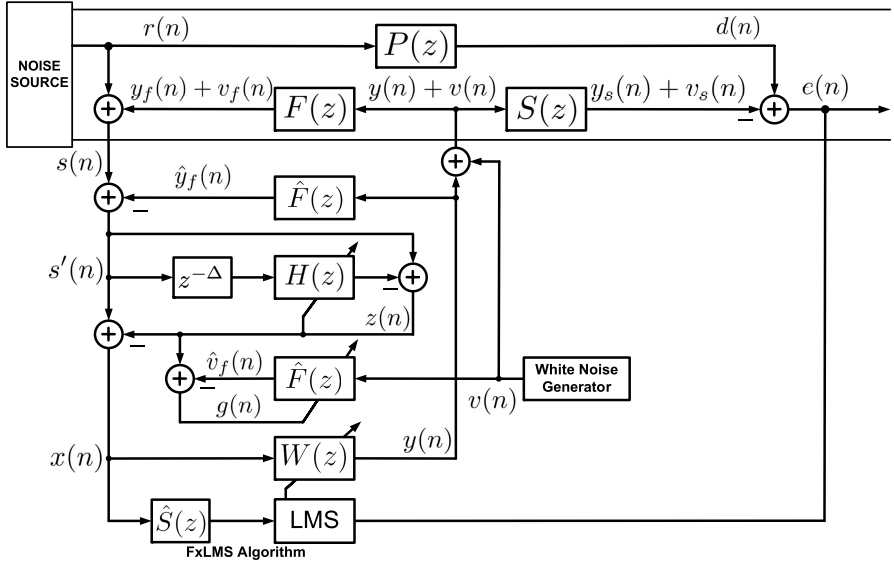

(a)

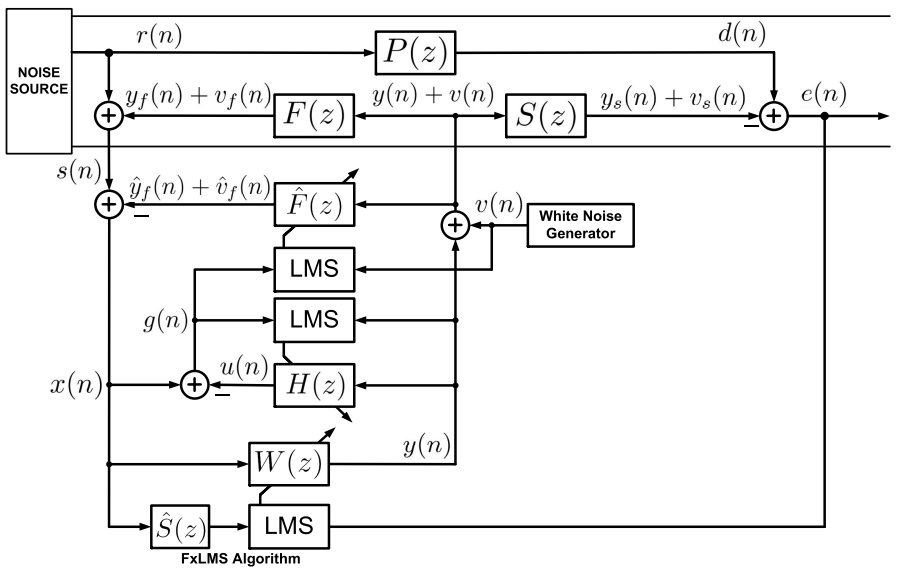

(b)

Fig. 2. Additive random noise based methods for online FBPM in ANC systems: (a) Kuo's method with with signal discrimination filter. (b) Proposed simplified method with adaptive noise cancelation (ADNC) filter.

the error signal as

$$
e(n)=d(n)-y_{s}(n)-v_{s}(n)
$$

and upstream to give corrupted reference signal as

$$
s(n)=r(n)+y_{f}(n)+v_{f}(n)
$$

where $d(n)=p(n) * r(n)$ is the primary noise signal; $y_{s}(n)=s(n) * y(n)$ is the antinoise signal; $v_{s}(n)$ is a component due to the modeling signal $v(n)$; $y_{f}(n)=f(n) * y(n)$ and $v_{f}(n)=f(n) * v(n)$ are the feedback components due to the canceling signal $y(n)$ and the modeling signal $v(n)$, respectively; $p(n), s(n), f(n)$ are impulse responses of $P(z), S(z)$, and $F(z)$, respectively; and $*$ denotes the linear convolution. The output of the $\operatorname{FBPMN} \hat{F}(z)$ filter is subtracted from $s(n)$ to generate the reference signal for $W(z)$ as

$$
x(n)=r(n)+\left[y_{f}(n)-\hat{y}_{f}(n)\right]+\left[v_{f}(n)-\hat{v}_{f}(n)\right] .
$$

The ANC filter $W(z)$ is adapted by FxLMS algorithm as

$$
\boldsymbol{w}(n+1)=\boldsymbol{w}(n)+\mu_{w} e(n) \boldsymbol{x}^{\prime}(n)
$$


where $\mu_{w}$ is the step size for $W(z), \boldsymbol{x}^{\prime}(n)=\left[x^{\prime}(n), x^{\prime}(n-1), \cdots, x^{\prime}(n-L+\right.$ 1) $]^{T}, L$ is tap-weight length of $W(z)$ and $x^{\prime}(n)=\hat{s}(n) * x(n)$ is the reference signal $x(n)$ filtered through $\hat{S}(z)$. The ADNC filter $H(z)$ takes $y(n)$ as its input, and $x(n)$ as its desired response to generate the error signal

$$
g(n)=r(n)+\left[y_{f}(n)-\hat{y}_{f}(n)\right]+\left[v_{f}(n)-\hat{v}_{f}(n)\right]-u(n)
$$

where $u(n)$ is the output of $H(z)$. The coefficients of $H(z)$ are updated by LMS algorithm

$$
\boldsymbol{h}(n+1)=\boldsymbol{h}(n)+\mu_{h} g(n) \boldsymbol{y}(n)
$$

where $\mu_{h}$ is step size parameter for $H(z), \boldsymbol{y}(n)=[y(n-1), y(n-2), \cdots, y(n-$ $M)]^{T}$, and $M$ is tap-weight length of $H(z)$. In $(7),\left[v_{f}(n)-\hat{v}_{f}(n)\right]$ is error signal required for adaptation of FBPMN filter $\hat{F}(z)$. The gradient in LMS equation for $\hat{F}(z)$ is computed using same error signal $g(n)$ and the random signal $v(n)$ as input signal, resulting in the following LMS update equation for $\hat{F}(z)$

$$
\hat{\boldsymbol{f}}(n+1)=\hat{\boldsymbol{f}}(n)+\mu_{f} g(n) \boldsymbol{v}(n) .
$$

Assuming that $H(z)$, and $\hat{F}(z)$ have converged; $u(n) \rightarrow r(n)+\left[y_{f}(n)-\hat{y}_{f}(n)\right]$, $\hat{v}_{f}(n) \approx v_{f}(n)$ and $\hat{y}_{f}(n) \approx y_{f}(n)$. Thus input to $W(z)$ is $x(n) \approx r(n)$, and is free of any noise.

\subsection{Computational Complexity}

A computational complexity analysis shows that, in comparison with the previous methods, the proposed method requires $N$ less multiplications per iteration and $N-1$ less additions per iteration, where $N$ is tap-weight length of $\hat{F}(z)$. This is advantageous when length of the adaptive filter is long, which is usually the case in practical ANC systems.

\section{Computer Simulations and Concluding Remarks}

This section presents the simulation experiments performed to verify the effectiveness of the proposed method. Here we compare the performance of the proposed method with the Kuo's method [6], and authors' previous work [7]. For acoustic paths the experimental data provided by [1] is used. Using this data, $P(z), S(z)$ and $F(z)$ are modeled as FIR filters of tapweight lengths 48,16 , and 32 , respectively. The adaptive filter $W(z), H(z)$ and $\hat{F}(z)$ are FIR filters of tap-weight length $L=32, M=16$, and $N=32$, respectively. The decorrelation delay $\Delta$ in Kuo's method [6] is 30. All the adaptive filters are initialized by null vectors of an appropriate order. A sampling frequency of $2 \mathrm{kHz}$ is used. The reference signal $x(n)$ comprises sinusoids of 150,300 and $450 \mathrm{~Hz}$ and a zero-mean white noise is added with SNR of $30 \mathrm{~dB}$. The modeling excitation signal $v(n)$ is a zero-mean white Gaussian noise of variance 0.05. The step size parameters are adjusted for fast and stable convergence, and, by trial-and-error, are found to be, $\mu_{w}=$ $1 \times 10^{-5}, \mu_{f}=5 \times 10^{-3}, \mu_{h}=5 \times 10^{-4}$.

The simulation results are shown in Fig. 3. Figure 3 (a) shows the curves for relative modeling error $\Delta F$ being defined as $\Delta F(n)=(\|\boldsymbol{f}(n)-\hat{\boldsymbol{f}}(n)\| /$ 


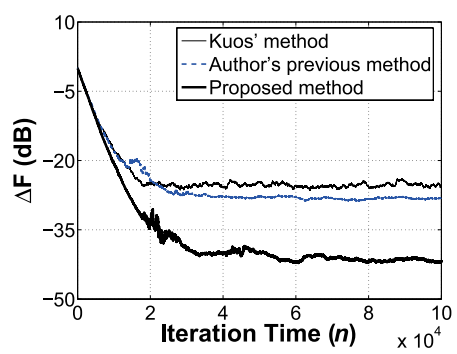

(a)

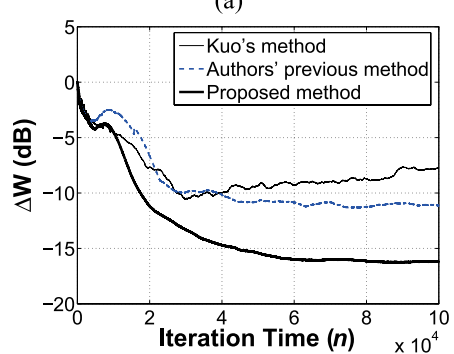

(b)

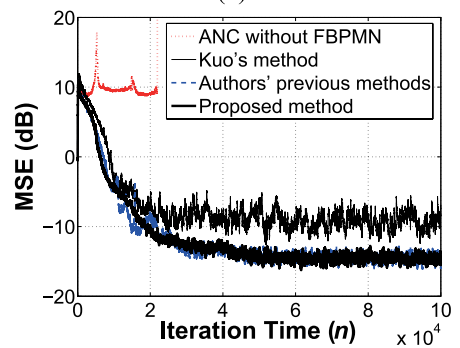

(c)

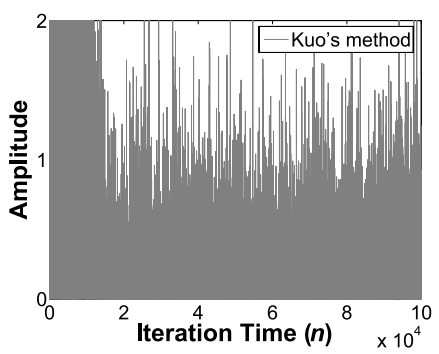

(d)

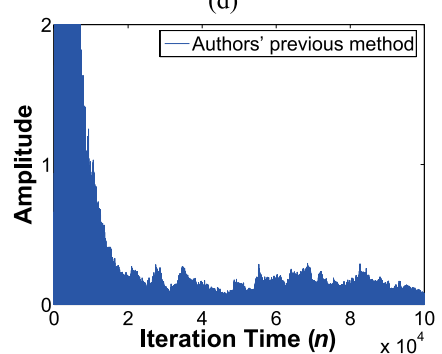

(e)

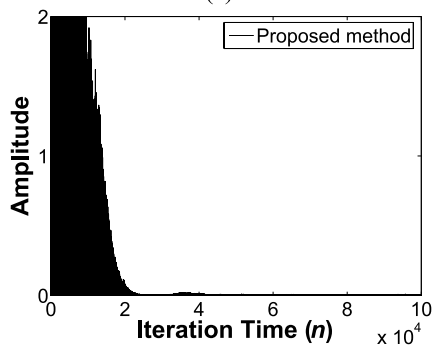

(f)

Fig. 3. (a) Curves for relative modeling error $\Delta F(\mathrm{~dB})$. (b) Curves for estimation error $\Delta W(\mathrm{~dB})$. (c) Curves for mean square error. d), e), and f) Curves for (squared) error in reference signal: $\Delta X(n)=[x(n)-r(n)]^{2}$.

$\|\boldsymbol{f}(n)\|)$. There curves are average of 10 realizations. We see that for same simulation parameters, the proposed method can reduce the relative modeling error to a lower level. Figure 3 (b) shows the curves for the estimation error for $W(z)$ being defined as $\Delta W(n)=\left(\left\|\boldsymbol{w}(n)-\boldsymbol{w}^{\circ}(n)\right\| /\|\boldsymbol{w}(n)\|\right)$, where $\boldsymbol{w}^{\circ}(n)$ is the optimal value of the weight vector of the ANC controller that is obtained by using FxLMS algorithm under no acoustic feedback condition. Again proposed method achieves better performance than the other methods. The corresponding curves for mean square error are shown in Fig. 3 (c). We see that, without FBPMN, the ANC system becomes unstable. Kuo's method and Author's method can stabilize the ANC system, with Author's method giving better performance. Figures (3)(d)-(f) show curves for (squared) error in the reference signal $x(n)$, for single realization. It is evident that proposed method is better able to remove the acoustic feedback, than the other methods.

In conclusion, here we have presented a simplified version of our previous method for acoustic feedback neutralization in ANC systems. We have demonstrated through the computer simulations, that the proposed method gives improved performance, and at somewhat reduced computational cost. 